\title{
Outcomes of Renal Function in Cardiogenic Shock Patients With or Without Mechanical Circulatory Support
}

\author{
Vandan D. Upadhyaya ${ }^{\mathrm{a}, \mathrm{d}}$, Abbas Alshami ${ }^{\mathrm{a}}$, Ishan Patel ${ }^{\mathrm{a}}$, Steven Douedi ${ }^{\mathrm{a}}$, Amy Quinlan ${ }^{\mathrm{b}}$, \\ Tresy Thomas ${ }^{c}$, Joni Prenticec ${ }^{c}$, Dawn Calderon ${ }^{c}$, Arif Asif ${ }^{a}$, Shuvendu Sen ${ }^{a}$, \\ Aditya Mehrac ${ }^{c}$, Mohammad A. Hossain ${ }^{\mathrm{a}}$
}

\begin{abstract}
Background: The objective of the study was to compare the renal outcomes in patients presenting with all-cause cardiogenic shock who were supported by either Impella devices (Abiomed, Danvers, MA), intra-aortic balloon pump (IABP), or vasopressors alone. Outcomes of cardiogenic shock remain poor even with the advancement of early revascularization and circulatory supportive care. Percutaneous mechanical circulatory support (MCS) device has emerged as an effective strategy in protecting end organ function especially renal function during high risk percutaneous coronary intervention (PCI) and in patients with cardiogenic shock. Currently, comparative data amongst various MCS modalities and their association with improvement of renal function in cardiogenic shock patients have not been well characterized.
\end{abstract}

Methods: Data from New Jersey Cardiac Catheterization Data registry of cardiogenic shock patients from a single tertiary care institution that underwent cardiac catheterization and the modality used to treat were obtained, either with Impella devices, IABP, or treatment with vasopressors alone. Retrospective chart review was conducted to assess the incidence of acute kidney injury (AKI) on patients with cardiogenic shock prior to and after cardiac catheterization and renal function was evaluated over the course of $96 \mathrm{~h}$ after cardiac catheterization. Statistical analysis was performed to ascertain significant difference in creatinine and estimated glomerular filtration rate (eGFR) in patients who received Impella devices, IABP, or were treated with vasopressors alone.

Results: A total of 61 all-cause cardiogenic shock patients met the inclusion and exclusion criteria and were included in the study with

Manuscript submitted February 11, 2021, accepted May 12, 2021

Published online May 25, 2021

aDepartment of Medicine, Hackensack Meridian Jersey Shore University Medical Center, Neptune, NJ 07753, USA

${ }^{b}$ Department of Cardiology, One Robert Wood Johnson Place, New Brunswick, NJ 08903, USA

'Department of Cardiology, Hackensack Meridian Jersey Shore University Medical Center, Neptune, NJ 07753, USA

${ }^{\mathrm{d} C}$ Corresponding Author: Vandan Upadhyaya, Department of Internal Medicine, Hackensack Meridian Jersey Shore University Medical Center, 1945 Route 33, Neptune, NJ 07753, USA. Email: Vandan.upadhyaya@hmhn.org

doi: https://doi.org/10.14740/jocmr4449
19 receiving IABPs, 15 receiving Impella devices, and 27 treated with vasopressors alone. Baseline characteristics among these three groups did not show any statistically significant difference. A total of 29 cardiogenic shock patients had experienced AKI prior to cardiac catheterization in which those receiving Impella devices showed statistically significant decrease in creatinine and increase in eGFR at 72 and $96 \mathrm{~h}(\mathrm{P}<0.05)$ compared to baseline. Within the same cohort, Impella group showed statistically significant lower creatinine at $96 \mathrm{~h}$ when compared to IABP. Patients that experienced AKI after cardiac catheterization did not show any statistically significant changes in renal function regardless of modality used.

Conclusion: The results of our study suggest that Impella devices improve renal function in all-cause cardiogenic shock patients who experience AKI prior to undergoing cardiac catheterization.

Keywords: Acute kidney injury; Cardiogenic shock; Intra-aortic balloon pump; Impella; AMI-CS; Mechanical circulatory support

\section{Introduction}

Despite advances in rate of early revascularization, the prognosis of patients with cardiogenic shock remains poor [1]. Multisystem organ failure because of systemic hypoperfusion is known to occur in more than $50 \%$ of patients with cardiogenic shock, and due to its sensitivity to poor perfusion, one of the first organs to suffer in cardiogenic shock are the kidneys [2]. Acute kidney injury (AKI) is also one of the serious complications after cardiac procedures and is associated with high postoperative morbidity and mortality [3]. Percutaneous mechanical circulatory support (MCS) devices are beneficial for patients with cardiogenic shock, specifically those undergoing complex coronary revascularization [1].

Percutaneous MCS has emerged as an effective strategy in protecting end organ function during high risk percutaneous coronary intervention (PCI) [4]. Modalities of percutaneous MCS exist based on their different distinct hemodynamic profile, but all available devices to a variable degree, improve cardiac output and blood pressure [1, 2, 4-6]. Intra-aortic balloon pump (IABP) counter pulsation therapy is used in patients with cardiogenic shock with the goal of decreasing myocardial oxygen consumption, increasing coronary artery perfusion, de- 
creasing afterload and enhancing cardiac output $[1,5]$. Similarly, Impella devices (Abiomed, Danvers, MA) are another form of MCS device that use non-pulsatile, axial flow pump that provides support by pumping blood from the left ventricle into the ascending aorta $[1,6-8]$.

Impella supported PCI has shown to decrease ischemic kidney injury from episodic hypoperfusion, whereas conflicting reports exist regarding the existence of improvement of renal function with the use of IABP [4-6]. In the setting of cardiogenic shock in patients who underwent PCI/coronary artery bypass graft $(\mathrm{CABG})$ with complex coronary revascularization, there are little comparative data amongst different MCS modalities displaying superiority in improvement of renal status. In addition, there is variability amongst studies on MCS and their effect on AKI because of inconsistent definitions of AKI and renal insufficiency [7-10].

We aimed to study patients with cardiogenic shock status post $\mathrm{PCI} / \mathrm{CABG}$ with or without AKI treated with IABPs, Impella devices, and those without MCS treated with vasopressors alone. The purpose of this study was to compare the outcome of renal function in this cohort using the widely accepted Kidney Disease: Improving Global Outcomes (KDI$\mathrm{GO}$ ) guidelines. We hypothesized that the cohort treated with Impella devices will have the most statistically significant improvement in renal function if they sustained AKI.

\section{Materials and Methods}

We sought the Strengthening the Reporting of Observational Studies in Epidemiology (STROBE) guidelines to report this study [11].

\section{Study design and collection of data}

The study was conducted at Hackensack Meridian Health Jersey Shore University Medical Center (JSUMC), Neptune, NJ, USA. Data were obtained from New Jersey Cardiac Catheterization registry for patients who have undergone cardiac catheterization at JSUMC in 2018 and 2019. The study was approved by the Institutional Review Board of Hackensack Meridian Health (study number: 201911091J-JSUMC). Data were analyzed retrospectively from medical records at JSUMC. Chart review was performed by using patient identifiers (first name, last name, date of birth, and medical record number) to obtain data based on the inclusion and exclusion criteria of this study. Data collection and organization was conducted with Microsoft excel software. The investigators had full access to these data and had control of the data analysis. All study procedures were carried out in accordance with the Declaration of Helsinki regarding research involving human subjects.

\section{Definitions}

Characteristics of the patients were defined as per New Jersey Cardiac Catheterization Registry (Supplementary Material 1, www.jocmr.org). AKI was defined per the KDIGO definition as an increase in serum creatinine by $>0.3 \mathrm{mg} / \mathrm{dL}$ within $48 \mathrm{~h}$ or increase in serum creatinine to $>1.5$ times baseline, which is known or presumed to in the last 7 days [12]. Baseline creatinine was defined to be the lowest creatinine value charted during the most recent prior admission. For patients without prior admissions, baseline creatinine was defined as the creatinine at the time of admission. Urine volume over every $6 \mathrm{~h}$ was not adequately recorded for every patient included in this study and thus was not used as a criterion for AKI. Estimated glomerular filtration rate (eGFR) was based on the Chronic Kidney Disease Epidemiology (CKD-EPI) collaboration formula as opposed to the Modification of Diet in Renal Disease Study equation to have a better estimation of GFR in cohort with normal or near-normal GFR [13].

\section{Inclusion and exclusion criteria}

Over the 2-year period (2018 - 2019), all patients with cardiogenic shock within $24 \mathrm{~h}$ of admission that underwent cardiac catheterization at JSUMC were included. Patients were separated based on the device inserted during cardiac catheterization into three groups: those that received IABP, Impella devices, and those that were treated with vasopressors alone. Our exclusion criteria were as follows: 1) those that underwent multiple cardiac catheterization in less than $96 \mathrm{~h}$; 2) patients who had received the device for less than $24 \mathrm{~h} ; 3$ ) any patient who experienced device malfunction; 4) any patient who died at the time of the procedure; 5) patients who were actively undergoing hemodialysis or required hemodialysis within $96 \mathrm{~h}$ after visit to the cardiac catheterization lab; 6) patients who were discharged from JSUMC early via transfers to another hospital in less than $96 \mathrm{~h}$; 7) those who had received multiple devices, switching from one device to another; 8) any patient that had a concurrent infection or charted diagnosis of "sepsis" at any time during the admission in which a device was placed; and 9) documented prior history of renal failure or if the patient has a history of creatinine $>2.0$.

\section{Study end points, variables, and statistics}

Using the New Jersey Cardiac Catheterization Registry, data of patients with cardiogenic shock within $24 \mathrm{~h}$ of admission, during which they underwent catheterization were obtained. Retrospective data collection was performed to obtain baseline creatinine, creatinine level prior to cardiac catheterization, and creatinine at 24,48 , and $96 \mathrm{~h}$ after cardiac catheterization. For each respective creatinine level, a corresponding eGFR was calculated using CKD-EPI equation. Chart review was conducted to obtain the modality through which cardiogenic shock was treated: IABP, Impella devices, or with vasopressors alone. Incidence of AKI, based on KDIGO definition, was noted based on when it occurred, either prior to cardiac catheterization or after cardiac catheterization. Based on the incidence of AKI, renal function, using creatinine and eGFR, was analyzed for $96 \mathrm{~h}$ and patients were differentiated based on the modality of treatment for un- 
derlying shock. Continuous and categorical variables were described as means and frequencies, as appropriate. All variables were tested for normal distribution with the Shapiro-Wilk test. Chi-square test was used to compare categorical variables. Paired sample Student's $t$-test was used to compare means of related samples, and independent sample Student's $t$-test was used to compare means of independent samples. An alpha (P) level of 0.05 was used to ascertain statistical significance. All analyses were done using IBM Statistical Package for the Social Sciences (SPSS) version 26.0 (IBM Corporation, Artmonk, NY).

\section{Results}

\section{Patient characteristics}

Demographics and characteristics of patients who were included in the study are shown in Table 1. A total of 61 cardiogenic shock patients met our inclusion and exclusion criteria. The median age among all 61 patients included in the study was 70.03 years, with 37 patients being males $(60 \%)$. Of the 61 patients, 19 had received IABP, 15 had received Impella devices, and 27 were treated with vasopressors alone. There was no statistically significant difference in the baseline patient characteristics amongst the three modalities of treatment. As per the exclusion criteria, no patients in this study were present with prior history of renal failure or prior dialysis. Of the 61 patients, 31 patients exhibited subjective symptoms compatible with an acute coronary syndrome (ACS) within $12 \mathrm{~h}$ of presentation, and there was no statistically significant difference amongst the incidence of symptoms and associated modalities of treatment for the associated cardiogenic shock $(\mathrm{P}=0.857)$. Most patients that presented with subjective symptoms correlating with ACS had associated ST-segment elevation on electrocardiogram $(\mathrm{n}=$ 27). There was no statistically significant difference $(P=0.961)$ in the incidence of ST-segment elevation and the method used for the treatment of cardiogenic shock.

\section{Use of vasopressors and/or inotropes}

Use of vasopressors and inotropes was categorized based on when the agents were first initiated in relation to the catheterization lab visit: initiation prior to catheterization lab, during the lab visit, or after the lab visit. The number of vasopressors or inotropes used, and the duration of use were unable to be obtained. Table 2 highlights the initiation of vasopressor and/or inotrope therapy in patients that had received IABP, Impella devices, or were treated with vasopressors alone. There was no statistically significant difference in the frequency or timing of vasopressor used in the different methods for treatment of cardiogenic shock.

\section{AKI prior to cardiac catheterization}

A total of 29 cases developed AKI prior to going the lab. Of them, 11 patients received IABP during the cardiac catheterization lab visit, five received Impella device, and 13 did not require any device and were treated with vasopressors alone. Table 3 shows serum creatinine means for the three groups over 96 h. Even though all $(n=5)$ AKI patients who received Impella devices had vasopressors initiated either prior or during the catheterization lab visit, as opposed to $63 \%(n=7)$ in the IABP group, the Impella group showed persistent decrease in creatinine (statistically significant only at 72 and 96 h). Impella group showed statistically significant lower creatinine at $96 \mathrm{~h}$ when compared to IABP. The IABP group showed statistically non-significant worsening of serum creatinine, and the group treated with only vasopressors showed statistically nonsignificant improvement (Fig. 1). Table 4 and Figure 2 show the mean eGFR of the three groups over $96 \mathrm{~h}$. Similarly, AKI patients receiving Impella devices showed statistically significant improvement in eGFR at 48, 72, and $96 \mathrm{~h}$ from baseline $(\mathrm{P}<0.05)$. IABP showed statistically significant worsening eGFR when compared to use of vasopressors alone at 48, 82, and $96 \mathrm{~h}(\mathrm{P}>0.05)$. Unlike creatinine, eGFR of patients with Impella devices showed borderline insignificant improvement compared to treatment with IABP at the $96 \mathrm{~h}$ mark $(\mathrm{P}=0.07)$.

\section{AKI after cardiac catheterization}

From 32 patients who did not experience AKI prior to cardiac catheterization, eight patients developed AKI after lab visit $(25 \%)$. Only one patient $(12.5 \%)$ with IABP developed AKI after lab visit, as opposed to four out of 10 in the Impella group $(40 \%)$. However, the difference was not statistically significant $(P=0.38)$. Because there was only one patient having received IABP, paired sample Student's $t$-test could not be performed. In addition, paired Student's $t$-test comparing both Impella and vasopressors only group showed no statistically significant changes. Similarly, Table 5 and Figure 3 show mean eGFR for patients of three groups who endured AKI after cardiac catheterization. There was no statistically significant improvement in eGFR in any of the three groups.

\section{Serum creatinine in patients without incidence of AKI}

Table 6 shows the creatinine trends in patients who did not develop AKI. Patients receiving Impella devices persistently decreased the serum creatinine level from prior to catheterization over $96 \mathrm{~h}$ (statistically significant at 72 and $96 \mathrm{~h}$ ). Mean serum creatinine at $96 \mathrm{~h}$ was statistically lower in the Impella group than in the IABP group, despite no statistical difference prior to cardiac catheterization (Fig. 4). Table 7 and Figure 5 show eGFR in patients without AKI. Like changes in creatinine, despite not experiencing AKI, there was still statistically improvement in mean eGFR at 48, 72, and $96 \mathrm{~h}$. No statistically significant change in mean eGFR was seen in any group when compared to baseline or when compared to other modalities.

\section{Discussion}

Multiple trials have been performed to assess whether there is 


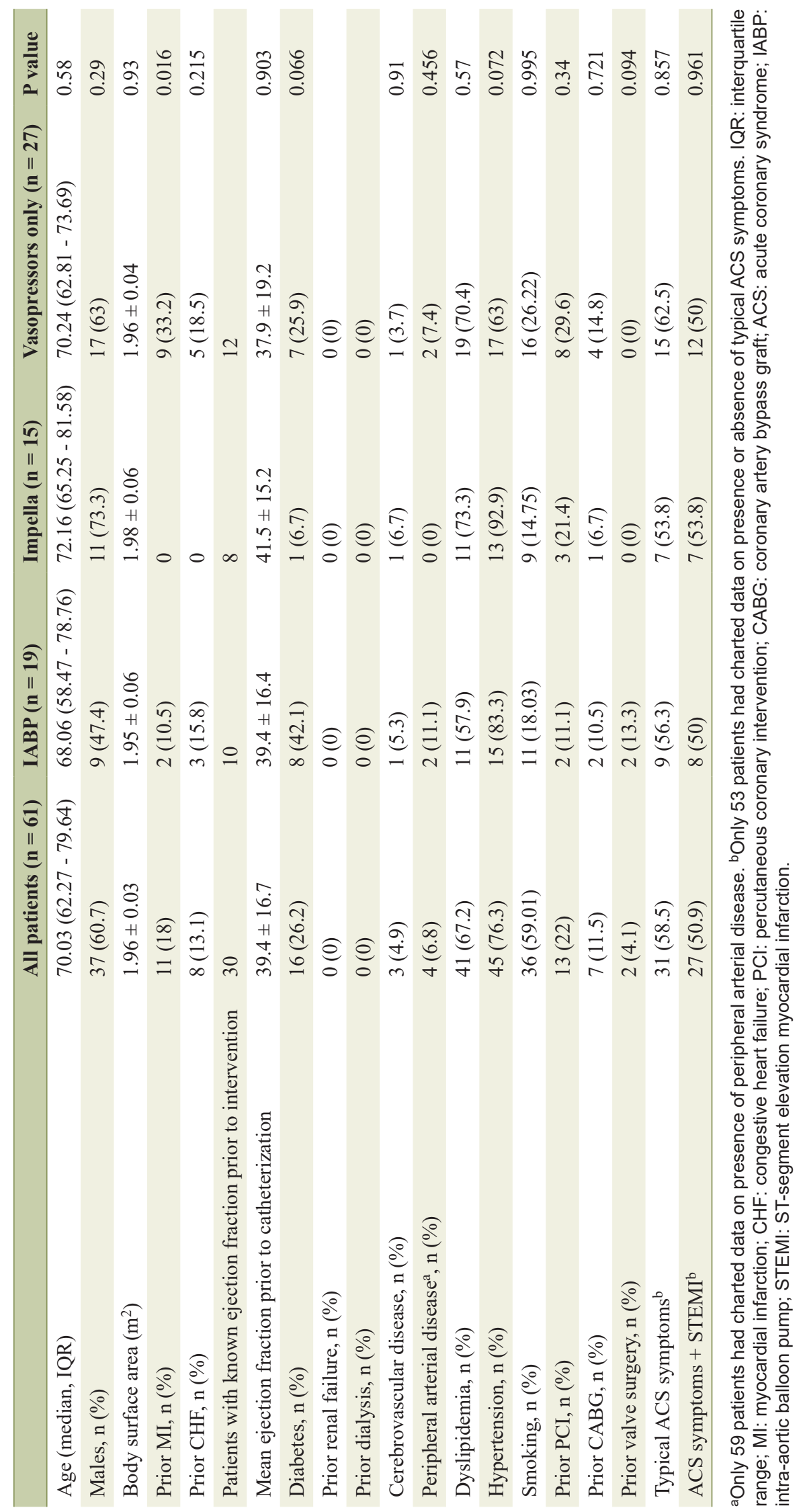


Table 2. Use of Vasopressors/Inotropes in Relation to Cardiac Catheterization Lab Visit

\begin{tabular}{|c|c|c|c|c|}
\hline Timing of vasopressor/inotrope use & $\operatorname{IABP}(n=19)$ & Impella $(n=15)$ & Vasopressors alone $(n=27)$ & $P$ value \\
\hline None use & 3 & 2 & 0 & 0.111 \\
\hline Use initiated before lab visit & 5 & 3 & 12 & 0.208 \\
\hline Use initiated after lab visit & 3 & 1 & 3 & 0.707 \\
\hline
\end{tabular}

IABP: intra-aortic balloon pump.

Table 3. Mean Serum Creatinine With Standard Errors for Patients With AKI Prior to Catheterization

\begin{tabular}{llllll} 
& Prior to catheterization & $\mathbf{2 4} \mathbf{h}$ & $\mathbf{4 8} \mathbf{h}$ & $\mathbf{7 2} \mathbf{h}$ & $\mathbf{9 6} \mathbf{h}$ \\
\hline Vasopressors alone & $1.54(0.07)$ & $1.29(0.15)$ & $1.32(0.28)$ & $1.27(0.27)$ & $1.20(0.2)$ \\
Impella & $1.64(0.22)$ & $1.42(0.15)$ & $1.38(0.29)$ & $1.21(0.27)^{\mathrm{a}}$ & $1.04(0.07)^{\mathrm{a}}$ \\
IABP & $1.68(0.21)$ & $1.90(0.23)^{\mathrm{b}}$ & $1.83(0.26)$ & $1.78(0.26)$ & $1.80(0.3)^{\mathrm{c}}$ \\
\hline
\end{tabular}

aStatistically significant from baseline (prior to catheterization). bStatistically significant in comparison with vasopressors alone group. cStatistically significant in comparison with Impella. AKI: acute kidney injury. IABP: intra-aortic balloon pump; AKI: acute kidney injury.

improvement in mortality in cardiogenic shock patients using percutaneous MCS devices. IABP-SHOCK II trial (Intra-aortic Balloon Pump in Cardiogenic Shock) is the first randomized controlled trial to assess the utility of IABP in patients with acute myocardial infarction complicated by cardiogenic shock (AMI-CS) [14]. The trial found no survival benefit for the use of IABP when compared with medical therapy. IMPRESS in Severe Shock trial (Impella Versus IABP Reduces Mortality in

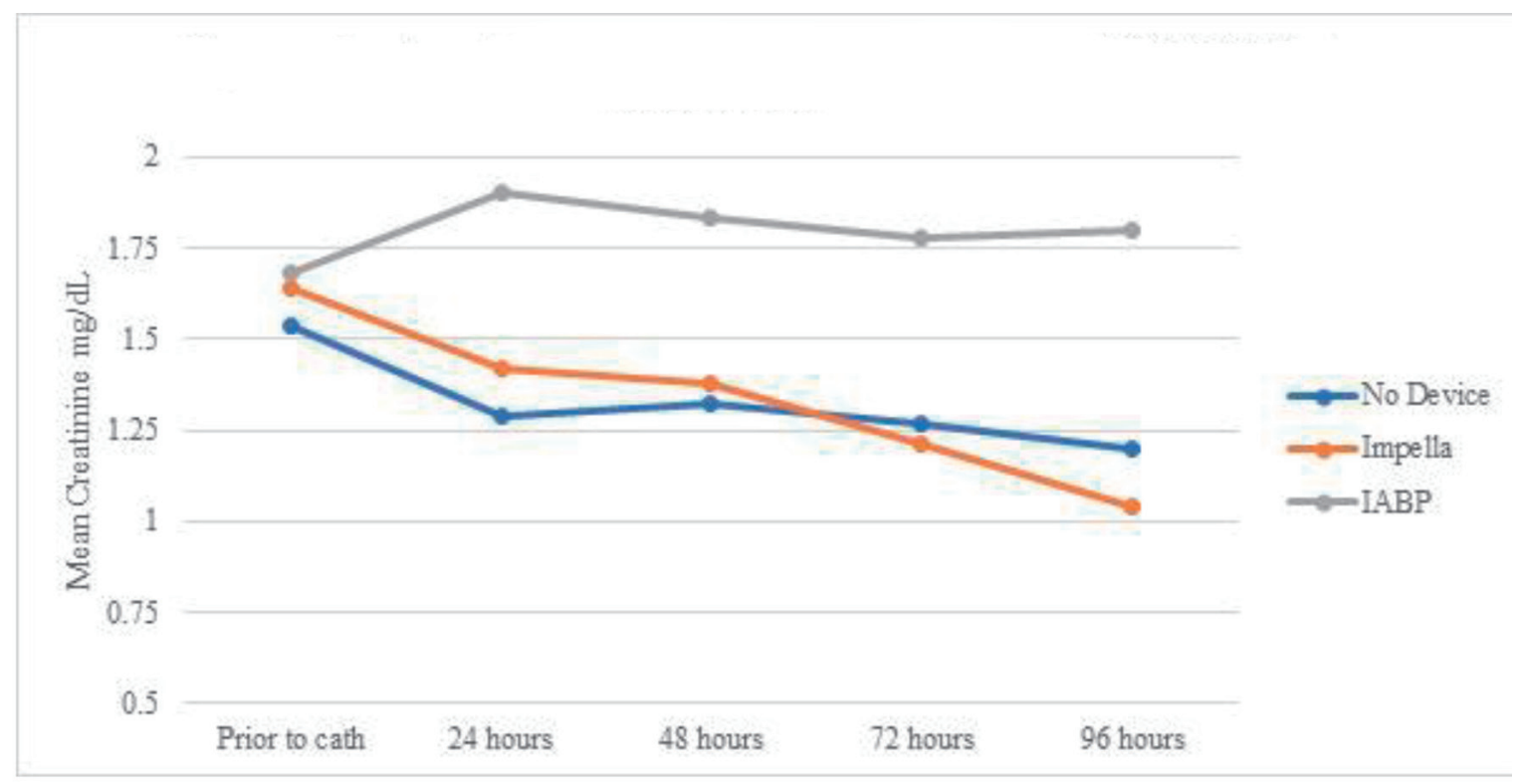

Figure 1. Mean serum creatinine over time by device for patients with AKI prior to cardiac catheterization. AKI: acute kidney injury; IABP: intra-aortic balloon pump.

Table 4. Mean eGFR With Standard Errors for Patients With AKI Prior to Catheterization

\begin{tabular}{llllll}
\hline & Prior to catheterization & $\mathbf{2 4} \mathbf{h}$ & $\mathbf{4 8} \mathbf{h}$ & $\mathbf{7 2} \mathbf{~ h}$ & $\mathbf{9 6} \mathbf{h}$ \\
\hline Vasopressors alone & $46.56(2.17)$ & $62.64(6)^{\mathrm{a}}$ & $69.29(7.29)^{\mathrm{a}}$ & $70.69(6.46)^{\mathrm{a}}$ & $73.60(6.14)^{\mathrm{a}}$ \\
Impella & $44.42(8.49)$ & $51.48(10.33)$ & $58.48(11.33)^{\mathrm{a}}$ & $69.28(13.54)^{\mathrm{a}}$ & $70.75(8.16)^{\mathrm{a}}$ \\
IABP & $43.71(7.96)$ & $37.9(7.10)^{\mathrm{b}}$ & $41(8.47)^{\mathrm{b}}$ & $43.42(9.63)^{\mathrm{b}}$ & $45.07(10.21)^{\mathrm{b}}$ \\
\hline
\end{tabular}

aStatistically significant from baseline. 'bStatistically significant in comparison with vasopressors alone group. eGFR: estimated glomerular filtration rate; AKI: acute kidney injury; IABP: intra-aortic balloon pump. 


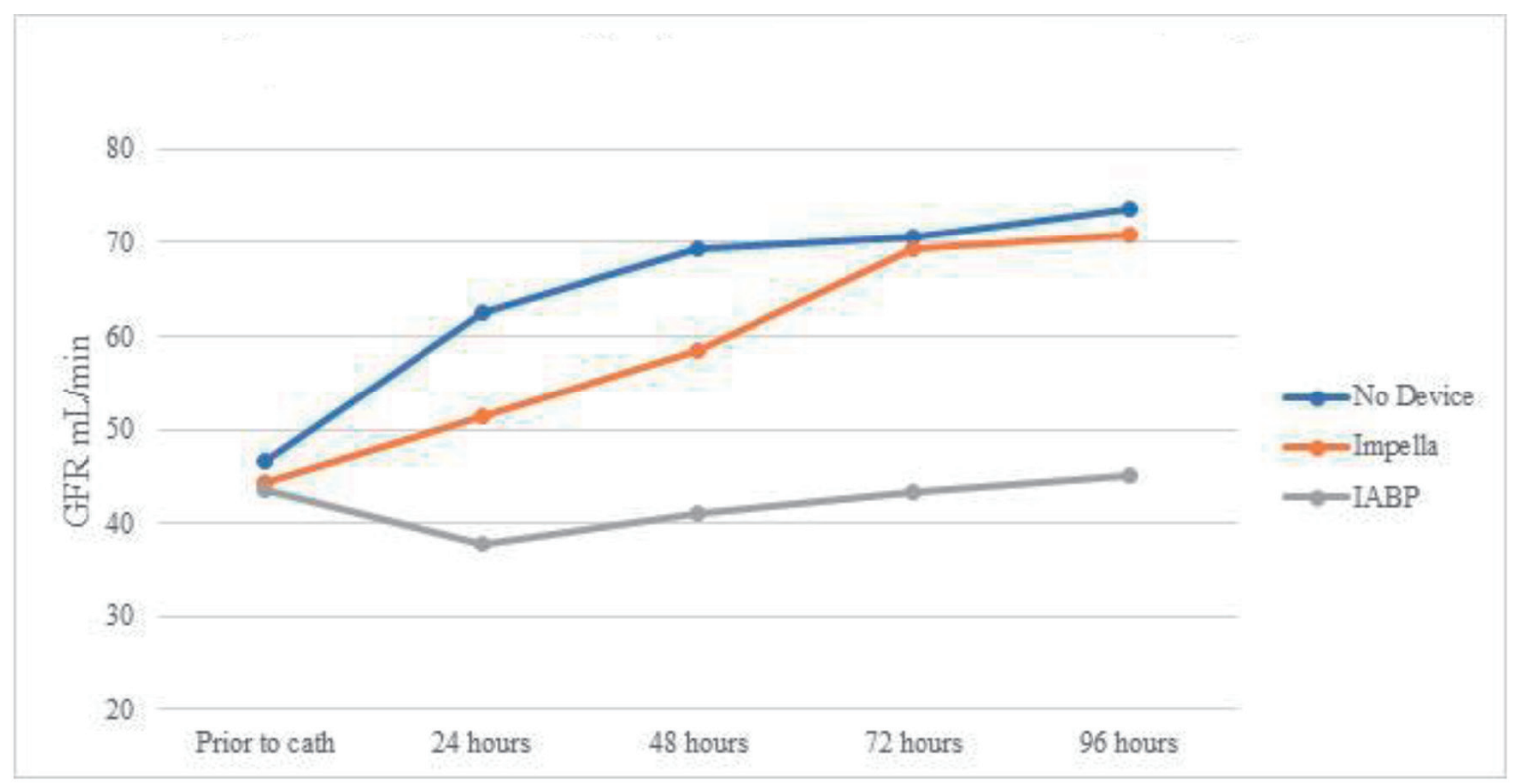

Figure 2. Mean eGFR over time by device for patients with AKI prior to cardiac catheterization. eGFR: estimated glomerular filtration rate; AKI: acute kidney injury; IABP: intra-aortic balloon pump.

Table 5. Mean eGFR With Standard Errors for Patients With AKI After Catheterization

\begin{tabular}{llllll}
\hline & Prior to catheterization & $\mathbf{2 4} \mathbf{h}$ & $\mathbf{4 8} \mathbf{h}$ & $\mathbf{7 2} \mathbf{~ h}$ & $\mathbf{9 6} \mathbf{h}$ \\
\hline Vasopressors alone & $59.51(7.09)$ & $35.57(3.71)^{\mathrm{a}}$ & $31.10(7.75)$ & $37.10(11.14)$ & $47.06(13.7)$ \\
Impella & $60.41(10.14)$ & $38.83(5.08)$ & $38.26(12.05)$ & $39.95(11.28)$ & $44.81(11.78)$ \\
IABP & 75.51 & 25.50 & 10.56 & 12.72 & 19.30 \\
\hline
\end{tabular}

aStatistically significant from baseline. 'S Since only IABP group included only one patient, no SE was reported. eGFR: estimated glomerular filtration rate; AKI: acute kidney injury; IABP: intra-aortic balloon pump.

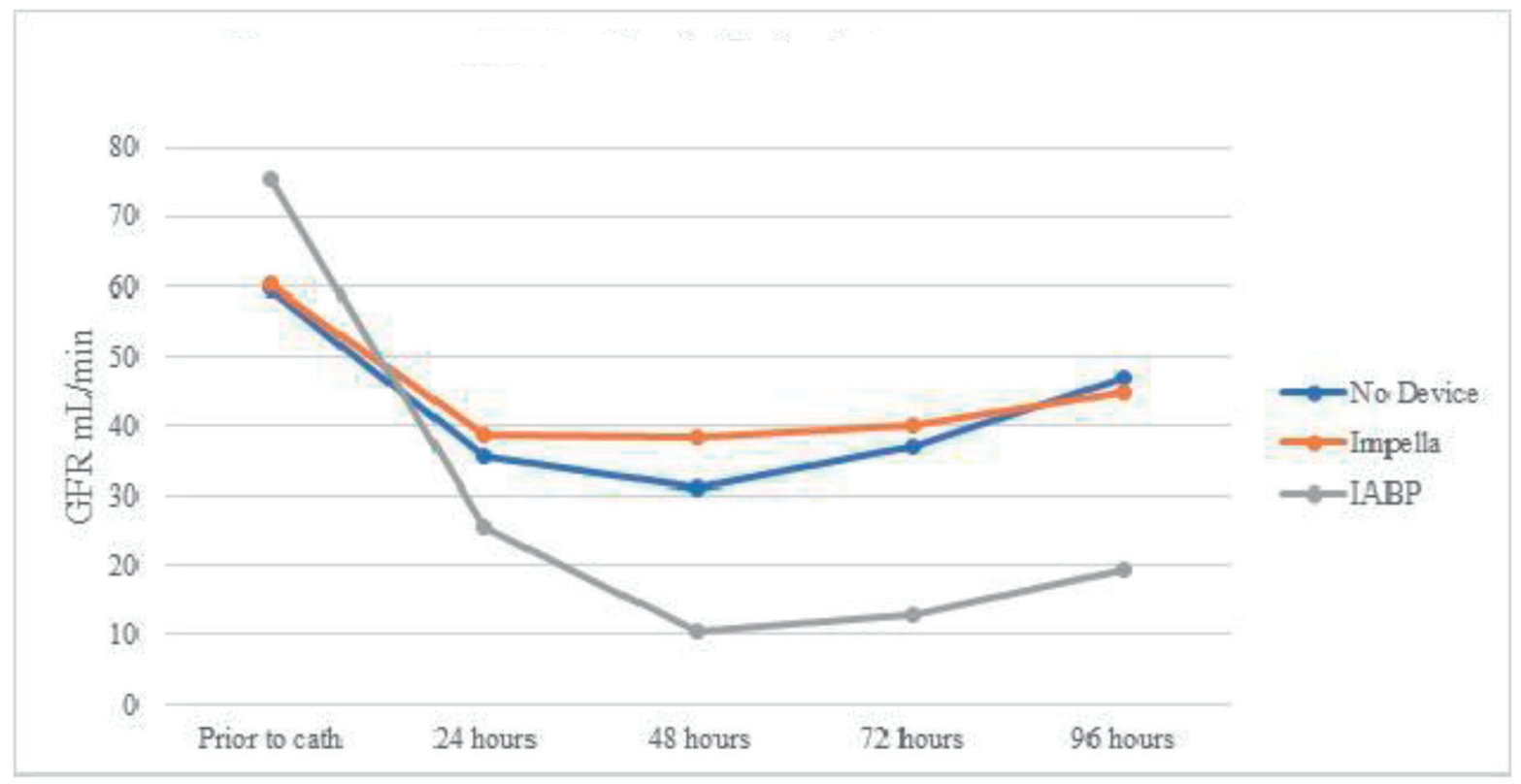

Figure 3. Mean eGFR over time by device for patients with AKI after cardiac catheterization. eGFR: estimated glomerular filtration rate; AKI: acute kidney injury; IABP: intra-aortic balloon pump. 
Table 6. Mean Serum Creatinine for Patients Without AKI

\begin{tabular}{llllll} 
& Prior to catheterization & $\mathbf{2 4} \mathbf{h}$ & $\mathbf{4 8} \mathbf{~ h}$ & $\mathbf{7 2} \mathbf{~ h}$ & $\mathbf{9 6} \mathbf{~ h}$ \\
\hline Vasopressors alone & $0.98(0.11)$ & $1.02(0.11)$ & $0.90(0.14)$ & $0.92(0.12)$ & $0.91(0.12)$ \\
Impella & $1.02(0.15)$ & $0.97(0.19)$ & $0.83(0.12)^{\mathrm{a}}$ & $0.75(0.10)^{\mathrm{a}}$ & $0.75(0.10)^{\mathrm{a}}$ \\
IABP & $0.96(0.05)$ & $0.87(0.04)$ & $0.91(0.08)$ & $0.86(0.10)$ & $0.92(0.08)$ \\
\hline
\end{tabular}

aStatistically significant when compared to prior to catheterization. AKI: acute kidney injury; IABP: intra-aortic balloon pump.

STEMI Patients Treated with Primary PCI in Severe Cardiogenic Shock) compared the use of Impella CP versus IABP in patients with AMI-CS and did not show any 30-day survival benefit in using one device over the other [15]. In a retrospective analysis of patients with AMI-CS, routine use of Impella devices at high-volume European centers as adjunctive therapy was compared to a similarly matched controlled group from the IABP-SHOCK II trial and found no significant difference in 30-day all-cause mortality with Impella as compared to IABP [16]. While differences in mortality currently are not seen when comparing IABP and Impella devices, some studies have found differences in adverse effects associated with their use. A large meta-analysis of four various randomized clinical trials performed by Rios et al in 2019 found that the use of peripheral left ventricular assist devices, including Impella devices, was associated with increased risk of combined short term adverse effects (AKI, limb ischemia, infection, major bleeding, and vascular injury) when compared to IABP (fixed effect relative risk (RR): 1.65, 95\% confidence interval (CI): 1.14 to $2.39 ; \mathrm{P}=0.008$ ) [17]. Conversely, observational data obtained from the prospective global catheter-based ventricular assist devices (cVADs) study by Flaherty et al showed that Impella-supported patients developed AKI at a much lower rate $(77.6 \%$ lower AKI risk at $48 \mathrm{~h} ; \mathrm{P}<0.001)$ during high-risk PCI compared to a predicted rate based on Mehran ContrastInduced Nephropathy Risk score model using the acute kidney injury network (AKIN) definition of AKI [18]. Their conclusion overall supported the findings that hemodynamic support with Impella was independently associated with reduction in the incidence of AKI during high-risk PCI.

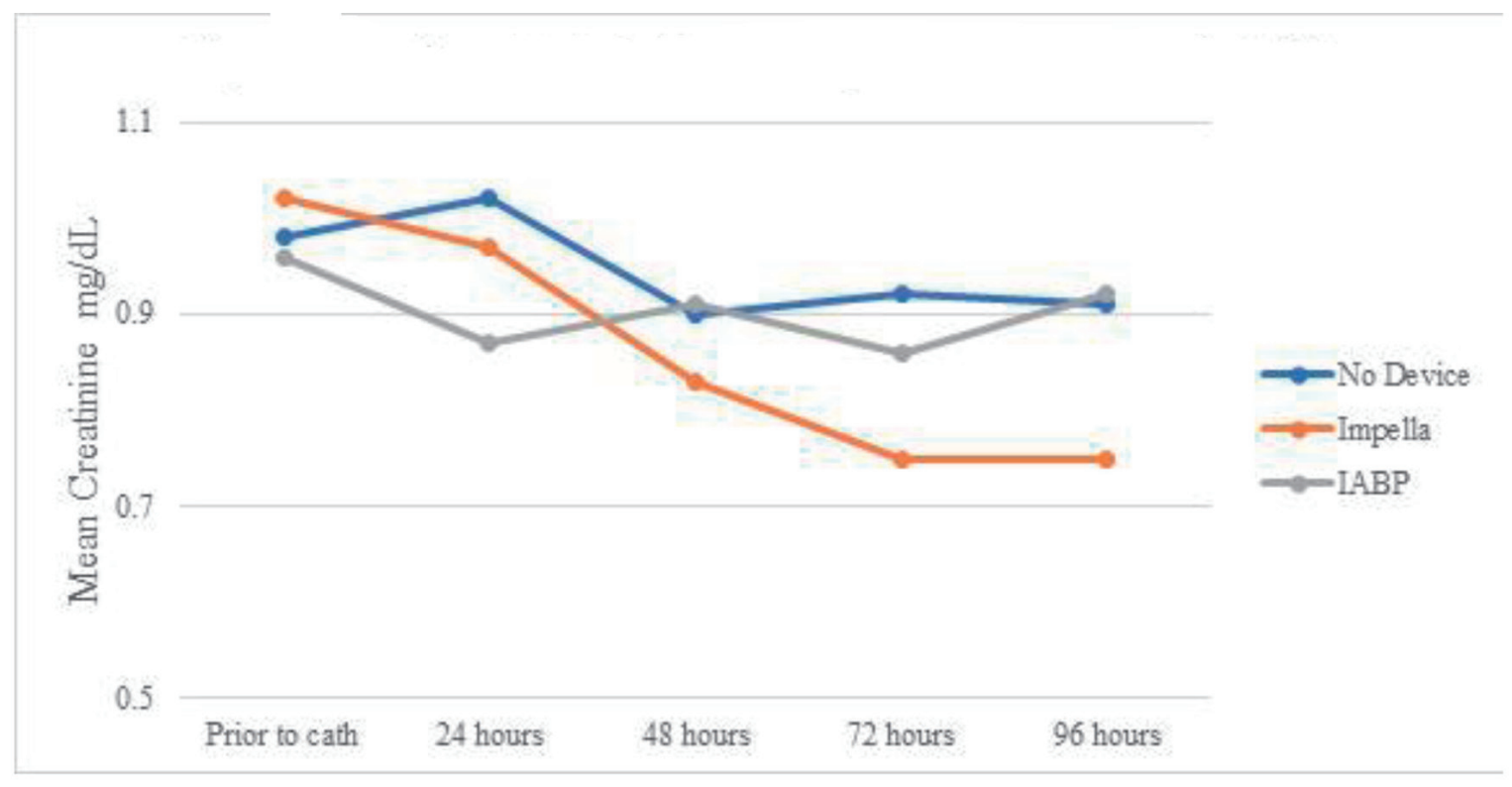

Figure 4. Mean serum creatinine over time by device for patients without AKI. AKI: acute kidney injury; IABP: intra-aortic balloon pump.

Table 7. Mean eGFR for Patients Without AKI

\begin{tabular}{llllll} 
& Prior to catheterization & $\mathbf{2 4} \mathbf{h}$ & $\mathbf{4 8} \mathbf{h}$ & $\mathbf{7 2} \mathbf{h}$ & $\mathbf{9 6} \mathbf{h}$ \\
\hline Vasopressors alone & $71.29(7.09)$ & $67.81(7.32)$ & $79.04(9.38)$ & $76.81(7.94)$ & $78.26(8.36)$ \\
Impella & $74.78(9.28)$ & $78.96(9.52)$ & $87.13(7.31)^{\mathrm{a}}$ & $91.96(4.92)^{\mathrm{a}}$ & $91.89(6.3)^{\mathrm{a}}$ \\
IABP & $68.97(4.22)$ & $76.44(5.57)$ & $75.26(7.40)$ & $81.04(8.37)$ & $77.49(7.84)$ \\
\hline
\end{tabular}

aStatistically significant when compared to baseline. eGFR: estimated glomerular filtration rate; AKI: acute kidney injury; IABP: intra-aortic balloon pump. 


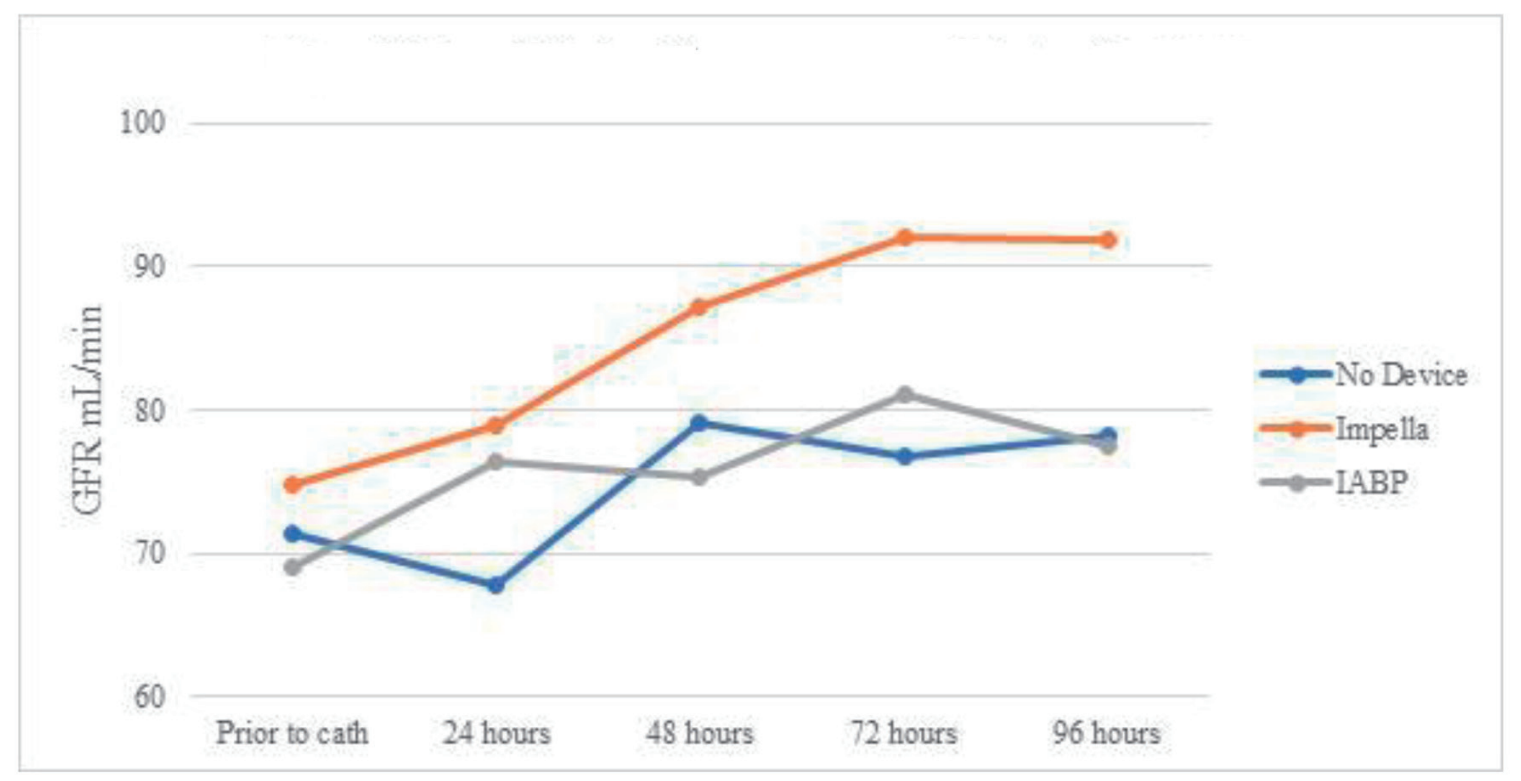

Figure 5. Mean eGFR over time by device for patients without AKI. eGFR: estimated glomerular filtration rate; AKI: acute kidney injury; IABP: intra-aortic balloon pump.

In our retrospective observational cohort study, we analyzed the outcomes of renal function by describing trends of creatinine and eGFR in cardiogenic shock patients with or without AKI that were treated with either IABP, Impella devices, or vasopressors alone. There was no statistically significant difference in the baseline characteristics, other than differences in prevalence of prior myocardial infarction, amongst those that were treated with IABP, Impella devices, or vasopressors alone. Those that received Impella devices were found to have statistically significant lower prevalence of myocardial infarction than those that were treated with IABP or vasopressors alone $(\mathrm{P}=0.016)$. There was no statistically significant difference in the frequency and timing of use of vasopressors amongst the cohort as well. A total of 61 patients met our inclusion and exclusion criteria, of whom 29 (48\%) patients experienced AKI prior to their visit to catheterization lab, eight (13\%) patients experienced AKI after cardiac catheterization, and 24 (39\%) patients never experienced AKI at all. We found statistically significant improvement in renal function after 72 and $96 \mathrm{~h}(\mathrm{P}<0.05)$, using creatinine and eGFR, in cardiogenic shock patients who experienced AKI prior to cardiac catheterization and received Impella devices. In this study, we report statistically significant improved renal function in patients with AKI prior to cardiac catheterization in the Impella group compared to those treated with IABP $(\mathrm{P}<$ $0.05)$. The study found that patients with AKI prior to cardiac catheterization that did not receive a device also had improvement in renal function overtime. While, definitively this relation cannot be confirmed, we feel that this cohort served as a control of patients who were deemed not to be as critically ill when compared to those that received a device. Operators inserted a device when a patient exhibited clinical and objective findings during the procedure that necessitated device insertion. Based on our findings, we validate the findings of
Flahtery et al in that Impella devices do show improvement in patients having sustained AKI [18]. Findings in our study do contradict the results of the meta-analysis performed by Rios et al: when compared with IABP, after $96 \mathrm{~h}$, our study does show statistically significant improvement of renal with the use of Impella devices when used to treat cardiogenic shock patients [17].

The results presented in this study may have significant implications. The presence of AKI during PCI or high-risk PCI is associated with increased mortality rates [19]. Inhospital mortality in AMI-CS patients with AKI not requiring dialysis was higher when compared to those without AKI (46\% vs. $34 \%$; odds ratio (OR): 1.67; 95\% CI: 1.65 - 1.69; $\mathrm{P}<$ 0.001 ) [20]. In the same study by Vallabhajosyula et al, AMICS patients with AKI had longer hospital stays, high adjusted total hospital costs, and had increased need of skilled nursing facilities after discharge [20]. While multi-center trials have reported no statistically significant difference in improvement of mortality when comparing Impella devices and IABP, there may be raising evidence of use of Impella in cardiogenic shock patients with AKI prior to cardiac catheterization as our study shows. Presence of AKI based on KDIGO definition or AKIN classification at the time of PCI or high-risk PCI, may be an indication to use one percutaneous device over another.

\section{Study limitations}

This study represents data from a single center experience over the course of 2 years, and conclusions from a limited sample size may be a limiting factor. Largest limiting factor associated with the study was that there was no standardized protocol for the treatment of patients with cardiogenic shock or AMI-CS patients at JSUMC during the time when the data were col- 
lected and thus patients may have received device support late as potentially lifesaving treatment. This study also did not control for the various types of Impella devices used as different devices may yield different results. Patients were also not controlled for post-PCI contrast nephropathy based on Mehran score as that may have also reflected on the incidence and improvement of AKI in this cohort. Because of the retrospective nature of the data collection, detailed data regarding amount contrast volume during cardiac catheterization or at the time of device insertion were not always available, thus the effect of contrast induced nephropathy in the outcome presented also is a confounding variable. Definitions from New Jersey Cardiac Catheterization Registry did not fall in accordance to similar variables used in large multicenter trials and thus comparative analyses amongst them may not be accurate. This study also did not include patients that required renal replacement therapy after cardiac catheterization as we felt this would confound the effect mechanical circulatory devices would have on GFR and creatinine. Further analysis would be helpful in patients with AKI after cardiac catheterization requiring renal replacement as it would be a crucial clinical outcome. This study also did not consider hemodynamic data of all the patients, and therefore there is lack of qualitative and quantitative data on the severity of cardiogenic shock that the cohort exhibited. This study also did not include cohort with device malfunction or device related mortality and associated renal dysfunction as etiology of malfunction or related mortality was not available.

\section{Conclusion}

In conclusion, the use of Impella devices in cardiogenic shock patients with AKI prior to cardiac catheterization is associated in improvement of renal function after $72 \mathrm{~h}$. This study shows that Impella devices exhibit greater improvement of renal function in this same cohort when compared to IABP after 96 $\mathrm{h}$. Such findings suggest implications of use of Impella devices over IABP in the treatment of cardiogenic shock patients with the presence of AKI prior to cardiac catheterization. Further students and adequately powered clinical trials are necessary to relate the efficacy of these devices.

\section{Supplementary Material}

Suppl 1. New Jersey Cardiac Catheterization Data Registry.

\section{Acknowledgments}

None to declare.

\section{Financial Disclosure}

The authors declare there was no funding obtained for this study.

\section{Conflict of Interest}

The authors declare that there is no relevant conflict of interest regarding the publication of this paper.

\section{Informed Consent}

Not applicable.

\section{Author Contributions}

VU, AA, SD, and IP were responsible for conceptualization, methodology, formal analysis, and writing of this paper. VU, IP, AQ, TT, and JP were responsible for obtaining NJ cardiac catheterization registry database, chart review, study design, and data collection. VU, AQ, AM, DC, AA, SS, and MH were responsible for obtaining institutional board review approval and data curation for this study. VU, AM, DC, AA, SS, and $\mathrm{MH}$ were responsible for reviewing and editing of this manuscript. All authors approved the submission of this manuscript for publication.

\section{Data Availability}

The authors declare that data supporting the findings of this study are available within the article.

\section{References}

1. Mandawat A, Rao SV. Percutaneous mechanical circulatory support devices in cardiogenic shock. Circ Cardiovasc Interv. 2017;10(5).

2. Abadeer AI, Kurlansky P, Chiuzan C, Truby L, Radhakrishnan J, Garan R, Topkara V, et al. Importance of stratifying acute kidney injury in cardiogenic shock resuscitated with mechanical circulatory support therapy. J Thorac Cardiovasc Surg. 2017;154(3):856-864 e854.

3. Tsai TT, Patel UD, Chang TI, Kennedy KF, Masoudi FA, Matheny ME, Kosiborod M, et al. Contemporary incidence, predictors, and outcomes of acute kidney injury in patients undergoing percutaneous coronary interventions: insights from the NCDR Cath-PCI registry. JACC Cardiovasc Interv. 2014;7(1):1-9.

4. Winkelmann ER, Steffens E, Windmoller P, Fontela PC, da Cruz DT, Battisti IDE. Preoperative expiratory and inspiratory muscle weakness to predict postoperative outcomes in patients undergoing elective cardiac surgery. J Card Surg. 2020;35(1):128-134.

5. Hilberman M, Derby GC, Spencer RJ, Stinson EB. Effect of the intra-aortic balloon pump upon postoperative renal function in man. Crit Care Med. 1981;9(2):85-89.

6. Flaherty MP, Pant S, Patel SV, Kilgore T, Dassanayaka S, Loughran JH, Rawasia W, et al. Hemodynamic support with a MICROAXIAL percutaneous left ventricular as- 
sist device (Impella) protects against acute kidney injury in patients undergoing high-risk percutaneous coronary intervention. Circ Res. 2017;120(4):692-700.

7. Dixon SR, Henriques JP, Mauri L, Sjauw K, Civitello A, Kar B, Loyalka P, et al. A prospective feasibility trial investigating the use of the Impella 2.5 system in patients undergoing high-risk percutaneous coronary intervention (The PROTECT I Trial): initial U.S. experience. JACC Cardiovasc Interv. 2009;2(2):91-96.

8. O'Neill WW, Kleiman NS, Moses J, Henriques JP, Dixon S, Massaro J, Palacios I, et al. A prospective, randomized clinical trial of hemodynamic support with Impella 2.5 versus intra-aortic balloon pump in patients undergoing high-risk percutaneous coronary intervention: the PROTECT II study. Circulation. 2012;126(14):1717-1727.

9. O'Neill WW, Schreiber T, Wohns DH, Rihal C, Naidu SS, Civitello AB, Dixon SR, et al. The current use of Impella 2.5 in acute myocardial infarction complicated by cardiogenic shock: results from the USpella Registry. J Interv Cardiol. 2014;27(1):1-11.

10. Shaw A, Stafford M, Swaminathan M. Role of biomarker in cardiac surgery in AKI. Year Book of Intensive Care and Emergency Medicine. 2009:14:612-619.

11. von Elm E, Altman DG, Egger M, Pocock SJ, Gotzsche PC, Vandenbroucke JP, Initiative S. The Strengthening the Reporting of Observational Studies in Epidemiology (STROBE) statement: guidelines for reporting observational studies. Ann Intern Med. 2007;147(8):573-577.

12. Khwaja A. KDIGO clinical practice guidelines for acute kidney injury. Nephron Clin Pract. 2012;120(4):c179184.

13. Levey AS, Stevens LA, Schmid CH, Zhang YL, Castro AF, 3rd, Feldman HI, Kusek JW, et al. A new equation to estimate glomerular filtration rate. Ann Intern Med. 2009;150(9):604-612.
14. Thiele H, Zeymer U, Neumann FJ, Ferenc M, Olbrich HG, Hausleiter J, Richardt G, et al. Intraaortic balloon support for myocardial infarction with cardiogenic shock. N Engl J Med. 2012;367(14):1287-1296.

15. Ouweneel DM, Eriksen E, Sjauw KD, van Dongen IM, Hirsch A, Packer EJ, Vis MM, et al. Percutaneous mechanical circulatory support versus intra-aortic balloon pump in cardiogenic shock after acute myocardial infarction. J Am Coll Cardiol. 2017;69(3):278-287.

16. Schrage B, Ibrahim K, Loehn T, Werner N, Sinning JM, Pappalardo F, Pieri M, et al. Impella support for acute myocardial infarction complicated by cardiogenic shock. Circulation. 2019;139(10):1249-1258.

17. Rios SA, Bravo CA, Weinreich M, Olmedo W, Villablanca P, Villela MA, Ramakrishna H, et al. Meta-analysis and trial sequential analysis comparing percutaneous ventricular assist devices versus intra-aortic balloon pump during high-risk percutaneous coronary intervention or cardiogenic shock. Am J Cardiol. 2018;122(8):1330-1338.

18. Flaherty MP, Moses JW, Westenfeld R, Palacios I, O'Neill WW, Schreiber TL, Lim MJ, et al. Impella support and acute kidney injury during high-risk percutaneous coronary intervention: The Global cVAD Renal Protection Study. Catheter Cardiovasc Interv. 2020;95(6):1111-1121.

19. Tsai TT, Patel UD, Chang TI, et al. Contemporary incidence, predictors, and outcomes of acute kidney injury in patients undergoing percutaneous coronary interventions: insights from the NCDR CATHPCI registry. JACC Cardiovasc Interv. 2014;7:1-9.

20. Vallabhajosyula S, Dunlay SM, Barsness GW, Vallabhajosyula S, Vallabhajosyula S, Sundaragiri PR, Gersh $\mathrm{BJ}$, et al. Temporal trends, predictors, and outcomes of acute kidney injury and hemodialysis use in acute myocardial infarction-related cardiogenic shock. PLoS One. 2019;14(9):e0222894. 